research that physiotherapists may well be interested in.

\section{COMMUNICATION IN THE RESEARCH ENVIRONMENT}

An important part of any research undertaking is the opportunity to discuss its value with one's peers. The MRC has a number of schemes to encourage communication amongst members of speciality groups and in this way foster co-operation and ensure that there is not a duplication of effort. For example, the MRC will support attendance at local conferences and assist scientists in this way to communicate with one another.

The MRC also provides the opportunity for scientists to apply for funds to spend a period of time at another research establishment within the country where a particular expertise is available. For example, one might wish to spend 3 months at a particular university working on a research project with physiotherapists of standing and this sort of visit could well be sponsored by the Medical Research Council.

The MRC also makes bursaries available for overseas study in a particular technique or area where South Africa does not have the necessary facilities. The question of the availability of similar facilities in South Africa is an important one, as the MRC must encourage the full utilization of its own scientific facilities before scientists go abroad to other establishments. So in effect we only support overseas visits if it involves something which cannot be done in South Africa and which has value for the South African situation. Scientists who obtain these overseas travel bursaries or overseas study bursaries, have to enter into an undertaking with the Medical Research Council to return to South Africa for a period of time so that what they have learned can be transferred to their colleagues in South Africa.

The MRC also supports the undertaking of international or national conferences. It does not, however, assist societies with their meetings; there are so many in the country that this would be a virtually impossible task. However, if an area of medicine warrants a scientific meeting and the research can clearly profit by the visit of overseas scientists to such a conference the MRC will support the undertaking financially and offer the facilities of its Conference Division for the organisation of such an MRC conference.

I hope from what I have said that you will nd believe that "research endeavours" will cure all the problems facing the physiotherapist. But certainly believe that it will offer an enriched career and im. proved treatment for the patient - an aim that everyone involved in health care shares!

\title{
THE NEUROSURGEON AND CHRONIC PAIN ${ }^{\dagger}$
}

\author{
J. C. DE VILLIERS* M.D., F.R.C.S. (Eng. \& Edin.)
}

The neurosurgeon is concerned predominantly with the management of chronic pain. His dealings with acute pain are similar to those of most other surgeons in that he attempts to remove the cause of the trouble, as in acute disc prolapse with back and leg pain.

Acute pain seems to serve a purpose. It causes avoidance of the injuring agent and induces rest. Some recurrent attacks of acute pain, however, may lead to a chronic disabjlity e.g. backache with or without sciatica. Some recurrent acute attacks of pain, such as in trigeminal neuralgia, serve no purpose and can drive the sufferer to suicide.

Patients with persistent pain can be divided into two categories: (a) Those with long-standing pain of continuous or recurring nature due to a lesion which is not life-threatening but in which the pain may threaten the patient's social integrity. (b) Those with severe pain of prolonged duration due to life-threatening lesions.

Whatever one believes about acute pain does not hold for chronic pain. Chronic unrelieved pain is a disease in itself. The patient does not adapt to it; on the contrary the sufferer seems to become sensitized. Lack of sleep, inability to concentrate and irritability are common associated features. The pain becomes the focal point of the patient's existence and he loses interest in everything else. He has seen many doctors, is disillusioned, disheartened and suspicious. Depression is a frequent feature of this state, the treatment of which can never be straightforward, simple or rapid.

* Helen and Morris Mauerberger Professor of Neurosurgery, University of Cape Town and Groote Schuur Hospital.

$\dagger$ Paper read at Pain Symposium, preceding 13th National Council Meeting 23-27 April 1979.

\section{THE PATIENT, THE PAIN AND THE PHYSICIAN}

It has been a traditional concept that surgeons should be concerned with the excision of lesions which are held to be useless or deleterious in their effects. Similarly it appeared logical that nerves, tracts and "centres" concerned with the transmission or interpretation of impulses concerned with the appreciation of pain should be divided or abated if such pain served no purpose. The history of surgery for pain shows that this attitude has been followed by disaster. Usually, when all surgery has failed, someor miraculously discovers that the patient is an individu with problems, worthy of consideration by a psychiatrist. All other "abstract" aids are then invoked to absolve the surgeon from any guilt feeling which he may have engendered as a result of having become part of the natural history of the patient's disease.

Any surgeon who embarks on surgery for chronic pain, without having spent time with his patient is set on a disaster course. Other people who will talk to the patient, no matter who they are - psychiatrists, psychologists, social workers, a nursing sister or paramedical staff - can learn about the patient and his reactions and should share their views with the surgeon; there is therefore everything to be said for a team approach.

The surgeon must get to know his patient and get to know him well and this knowledge can be gained only by repeated conversation in depth. An attempt should be made to establish the following points: What sensation does the patient call "pain"? How does he describe it in his own words without any provocation on the part of the examiner? What does this sensation, call it pain if you must, do to him, what does it do for him and what does he do with it? One 
should explore those reaches of conversation where one can form an inkling of what he expects or has expected from life. If his pain has been of long duration and due to a non-malignant condition, has he accepted the basic disability which has caused this discomfort?

That pain is not merely dependent on the intensity of stimulation of afferent nerve fibres, but is also profoundly influenced by psychological factors, has been shown by many workers. Many forebrain structures can influence the activity of ascending fibres in spinal cord and brain stem reticular formation. There is therefore considerable neurophysiological evidence for a central control of afferent input. In the "gate control" concept of the dorsal hom, it is believed that the rapidly conducting lemniscal system triggers this central inhibiting mechanism. There is ample evidence that these central mechanisms - physical and phsychological - play an important part, if not an all-important part, in pain experience. This may explain the conditioning treatment of painful states or the apparently excessive pain xperienced by anxious and excitable persons, a phenohenon with which we are all too familiar.

Pain is not an abstraction but requires a person to endure it and describe it; it cannot exist outside a person. This complaint is an interaction between cause, reception and reaction. There is no such thing as real pain and imaginary pain. Chronic pain is a total experience of a particular personality.

We do not experience pain at any one site in the nervous system but throughout its entire structure from the simplest sensory receptor to its "highest centres" in a complex interaction of intensity of stimulus and psychological reaction. Consequently it should not surprise us to find that persistent pain is often associated with a long history of pain, anxiety and depression, poor general health, resentment and lack of confidence in the doctor.

Pain is not an absolute quality. It fluctuates and can be influenced by the situations in which the patient finds himself. If he is intensely interested in something or is preoccupied with other problems, the pain tends to wane. This history, if elicited, is already of great assistance in the future management of the patient.

It would be totally wrong to leave the impression that all chronic pain is due to psychological factors. It would be equally foolish to regard chronic pain as having no psychological accompaniment.

Every neurosurgeon with experience in this field has 1 admit that there are, what one could call "painful seople". They have from an early life adopted the rôle of the sufferer for one reason or the other. Perhaps life was too painful for them! They bring their pain to the doctor and display it to him as any other person would show his collection of semi-precious stones, old books or stamps. This is done with a certain pride, which must obviously be subdued, and they have every reason to be proud. Have not many physicians and surgeons failed miserably, despite their eminence, in the presence of this extraordinary pain? They themselves are passive, gently smiling spectators of their own suffering and play little part in an attempt to alleviate it. No form of surgery will do these patients any good.

Before one considers any surgical or other destructive procedure for the relief of pain, it is mandatory to have a clear anatomical, pathological and personality diagnosis available. Any good neurosurgeon must have at least a moderate aversion to destructive surgery.

Any patient with chronic pain may develop new lesions which may be masked by his old complaint. An emotionally overactive or "neurotic" person has only the language of overreaction to tell of her symptoms even if these symptoms indicate organic disease. A patient may have had 3 operations on his back and the basic lesion may yet have been missed. One should have a healthy distrust of previous diagnoses of "functional state" and "hysterical pain", whatever that may mean.

\section{PROCEDURES AVAILABLE TO THE SURGEON}

Considering the procedures that can be used in treating pain, one can start with peripheral nervesection and end with various psychosurgical procedures with an endless variation in between. These procedures will be briefly mentioned with their indications and drawbacks.

\section{PROCEDURES DIRECTED TO THE PERIPHERAL NERVES}

\section{Peripheral Nerve Blocking}

Interruption of conduction in peripheral nerves by means of local anaesthetic or neurolytic agents plays an important part in pain relief, no matter whether one practices in a sophisticated hospital or in the bush.

\section{Local A naesthetic Injection}

Local anaesthetic injection provides analgesia in the area of distribution of the nerve which has been blocked. An interesting observation is that the pain relief may often outlast the duration of the pharmacological action of the blocking agent, probably because the nerve block interrupts the reflex reactions evoked by the noxious stimulus which maintains a vicious cycle.

Apart from its therapeutic effects a nerve block may be used for three reasons:-

It can help to establish the anatomical route by which the abnormal impulses are conducted, i.e. the nerve which is involved.

It can, to some extent, help to differentiate between different types of pain although this is less accurate; for instance, injecting a site where referred pain is felt will also relieve pain although one is not blocking the site of primary origin of the abnormal impulses.

It can serve a prognostic function in that it may indicate whether a section of a nerve will give relief. This is less useful than one would like it to be, because relief can be obtained from injecting a site of referred pain. It is perhaps most useful as a prognostic measure in that it allows one to observe the patient's reaction to the area of numbness which is produced by blocking. There are a small number of patients who react so adversely to the area of numbness that permanent nerve blocking cannot be tolerated.

Anaesthetic blocks work not only by interrupting pain pathways but also by reducing the total input of impulses which impinge on the $\mathrm{T}$ cells of the cord. The technique of nerve blocks will not be described but it must be stressed that the patient's co-operation and understanding are vital before one can expect any success with this kind of procedure.

\section{Chemical Neurolysis}

The use of chemical neurolytic agents usually produces a much longer pain relief which may range from weeks to months, although it is rare for it to last for longer than a few months.

Chemical neurolysis is much more difficult than local anaesthetic iniections because the placement of the needle must be far more accurate, as much smaller doses have to be used in order to avoid undesired side effects. 


\section{Peripheral Nerve Section}

Surgery for peripheral nerves is limited by the fact that axon fibres will inevitably regenerate and with this the original pain will return. In addition to this, most peripheral nerves are of a mixed variety which means that motor paralysis as well as sensory loss would follow section of such a nerve. This transgresses the basic principle of all destructive surgery, namely, that the latter should produce as little added deficit as possible. The presence of an anaesthetic patch may have a disadvantage, depending on where it is. There are also patients who become morbidly preoccupied with an area of numbness.

Nerve section is still done in occasional cases of single division trigeminal neuralgia but then only if there are contra-indications to a formal attack on the ganglion or root. There is also the not insignificant risk of so-called neuroma formation, which in its own right may be far more difficult to treat than the primary condition. This painful sequence of nerve section neuroma formation - repeated nerve section - more neuroma formation - higher nerve section ad infinitum is a dismal story which was often seen in the mismanagement of amputation stump pain in former times.

\section{Interruption of Autonomic Nerves}

After the great vogue for sympathectomy in the nineteen thirties and forties, the sympathetic system seems to have fallen into disfavour and its function as an afferent pathway has been disregarded or forgotten. It supplies a very wide field in the body and there are certain procedures directed at this system which have limited but highly effective results in pain relief. The autonomic afferents from the viscera are predominantly concerned with vasomotor tone and muscular contractility. Visceral pain is poorly localised, somewhat vaguely referred to the region of the organ involved but often more distantly felt. Because sympathetic nerves are poorly myelinated, sympathetic nerve block requires a low concentration of anaesthetic agent and a successful block does not bring about any hyperaesthesia or hypoaesthesia. If the latter do appear, it is due to coincidental involvement of somatic nerves in the vicinity. Successful sympathetic block is accompanied by local elevation in temperature, both subjective and objective, which may take some minutes before it can be registered; plethysmographically increased pulsation may be demonstrated. The part becomes dry because sweating is abolished and locally the veins are dilated. The most commonly used sympathetic blocks are those of the lumbar plexus, coeliac plexus and stellate ganglion.

Lumbar plexus blocks are done mostly for peripheral vascular disease involving the legs.

Coeliac plexus block can be a very effective method for the control of upper abdominal pain resulting from carcinoma of the pancreas or upper abdominal viscera. The coeliac ganglion and splanchnic nerves contain pain-mediating fibres from, as well as sympathetic fibres to, the upper abdominal viscera. If the disease is confined to the viscera and does not involve the abdominal wall, intercostal nerves or lumbar plexus, the pain may be relieved by coeliac block.

Sympathectomy can follow effective sympathetic block if the patients' general condition will allow surgery, which is usually fairly major and not without risk.

\section{Rhizotomy}

The one great advantage of posterior root section over peripheral nerve section is that it is not followed by axonal regeneration. On the strength of this, Abbe (1889) introduced this operation. It has the added ad. vantage that there is no associated motor paralysis Unfortunately, complete block of impulses at this level also interrupts proprioception and joint sensation; if extensive areas are involved, there may be skin damage due to loss of sensation. It is contra-indicated for the spinal roots of the brachial and lumbar plexus unless the limb is already useless owing to the primary disease. It has some application in the management of intractable pain due to malignant infiltration of tissues in the neck which cannot be treated in any other way. It has a limited success rate, probably much less than $60 \%$, and the cause of failure is attributed to the great overlap between adjacent nerve territories. Multiple root sections only partially overcome this problem and involve a fairly extensive operation to which one cannot submit very ill patients.

Dorsal rhizotomy is suitable for patients whose pain involves structures innervated by only one or two dorsal roots. The patient must be in good general condition to withstand the operation and have a reasor able life expectancy.

The one outstanding indication for root section is trigeminal neuralgia, in which it is successful in more than $90 \%$ of cases. It requires an open operation which is done through the middle fossa either intra. durally or extradurally, or from the posterior fossa. A combined root section of the 5 th- 10th and upper 3 or 4 cervical nerves can be an ideal operation for the relief of pain in the lower jaw and neck region due to infiltrative carcinoma of the floor of the mouth if the patient is fit enough to tolerate such surgery.

Controlled radio-frequency thermal destruction of the trigeminal root and ganglion, via an approach through the foramen ovale with a special electrode, may give excellent results in trigeminal neuralgia (Sweet and Wepsic 1974). Its advantage is that it does not require an open operation, and general superficial sensation to the face is spared. The only problem is that the recurrence rate of pain may be higher than with other procedures, but the procedure can be repeated.

\section{Chemical Rhizotomy}

By this method neurolytic solutions are introduced into the subarachnoid space and manipulated in such a way that only the nerve roots concerned are bathed in the destructive solution. In order to facilitate thif use is made of gravity control by utilising $5 \%$ phen in glycerin which is heavier than spinal fluid and with percolate down into the desired root sleeves (Maher 1955). This technique has been used particularly for the relief of cancer pain and in the treatment of spasticity and painful flexor spasms. It requires a meticulous technique; if this is strictly adhered to, there are very few complications. It is only when one uses higher concentrations of neurolytic agent and performs repeated or bilateral injections that problems occur. Unfortunately, the fear of complications has turned many doctors away from this very useful method of giving relief to patients for whom no other form of therapy is available.

\section{PROCEDURES ON THE SPINAL CORD}

\section{Antero-lateral Cordotomy}

Section of the spinothalamic tract was first performed by Martin in 1912 following a suggestion by Spiller in 1905 . The operation is usually done in the upper thoracic level, at about the level T.2, 3 for relief to be obtained unilaterally to the level of the 
umbilicus. Upper cordotomy will give $70 \%-75 \%$ pain relief. Complications occur in about $15 \%$ of individuals and when bilateral operations are done, the complication rate is even higher. Associated muscle weakness in the leg, loss of bladder and rectal control and impotence are some of the graver sequelae. Relief of pain can be highly effective but interference with the autonomic system may lead to an unstable blood pressure which has particular significance in the elderly or frail patient. The major drawback of this operation is the significant number of people who fail to benefit from it, or who after an initial successful result later relapse into pain again. Why this should happen is not clearly understood but there is no doubt that it occurs and one must assume that alternate pathways are opened up to conduct these impulses. There is also the problem of unpleasant dysaesthesia developing in the area of analgesia and this is proportional to the length of survival of the patient.

In summary it can be stated that it is a good operation for relief of strictly unilateral pain associated with malignant disease in patients with a limited life ixpectancy. It is not a very good operation for patients with chronic pain of non-malignant aetiology. If the level of analgesia is to include the upper abdomen and thorax a high cervical cordotomy has to be performed. This operation is, however, not to be urdertaken lightly as there is a much greater risk due to respiratory depression as well as the added loss of motor elements of the phrenic nerve. Effective analgesia of the fingers may lead to injury and chronic ulceration after minimal trauma. Another untoward feature is incapacitating dysaesthesia below the level of the shoulder and incomplete pain relief in the leg.

Open cordotomy, whether dorsal or cervical, demands a general anaesthetic and a period of postoperative recovery and may not be tolerated by debilitated people in whom it is indicated. It is an operation not without risk and the risk is proportional to the inexperience of the operator, because it is done on anatomical rather than functional grounds.

\section{Percutaneous Cordotomy}

High frequency coagulation of the ventro-lateral quadrant of the spinal cord utilising a unipolar necdle electrode which is introduced through the second cervical intervertebral foramen is a procedure which has become well-established since its introduction by Mullan et al. in 1965. It does not require a general anaesthetic or a post-operative period of recovery as is the case for open cordotomy and therefore becomes more applicable to the frail debilitated patient suffering from terminal cancer. The risks of the procedure are greatly reduced because the localisation of the lesion is dependent on functional testing by means of electrical stimulation so that muscular paralysis and other undesirable side effects can virtually be eliminated. It has the added advantage that it can be repeated if the initial result is not satisfactory. As with open cordotomy, risks of serious complications are greater if the operation is done bilaterally. It has a highly characteristic risk which is not present in the open thoracic procedure in that it creates a respiratory hazard in patients with limited respiraotry reserve. Even in patients with normal respiratory function, the bilateral operation has the characteristic problem of Ondine's curse i.e. the failure of respiratory drive when the patient falls asleep. It would seem that this operation had better not be done bilaterally unless it is absolutely indicated; if a second procedure is done, it should be done at a much lower level to eliminate the risk of respiratory failure. This operation also should be limited to patients with pain due to malignant disease and a life expectancy of not more than 6 months because of the high risk of unpleasant dysaesthesia. The patient with chronic pain not threatening life is not a candidate for this operation.

\section{Commissural Myelotomy}

This operation, which involves a median longitudinal section of the cord, has been performed in a sporadic way since 1926 . In the last few years it has gained in popularity again and can probably be done more accurately with the aid of the operating microscope. It has several advantages in the treatment of severe pain: (1) It has virtually no mortality. (2) It is effective in relieving bilateral pain in malignant disease. (3) There is no permanent disturbance of micturition, bowel function or motor power.

It has some drawbacks, however: (1) It is an open operation. (2) Immediately post-operatively transient but very severe dysaesthesiae and hyperalgesia may develop. (3) Proprioception is disturbed but this tends to improve. (4) There is a post-operative change in appreciation of pin-prick and temperature sensation in a band around the lower trunk and down the thighs. (5) Pain in the pelvis is not always relieved. (6) It is not indicated in non-malignant conditions.

\section{OPERATIONS ON THE BRAIN STEM}

\section{Medullary Spinothalamic Tractomy}

Theoretically, this operation would be expected to yield a higher anaesthetic level than a high percutaneous cervical cordotomy. There are two reasons that make it an unacceptable operation. It is a much more dangerous procedure and less accurate because general anaesthesia prevents simple physiological testing and verification of the position of the tract by these means. Evoked potentials could be used but this adds even more time to an operation primarly indicated for the seriously ill.

\section{Trigeminal Tractotomy}

Sjoqvist's operation, which divides the descending tract of the trigeminal for intractable facial pain, has been available since 1938 but has never gained great favour. The tract is not easily identifiable on the surface of the brain. If the incision is placed too far medially, it divides propriceptive fibres from the arm and if too far laterally, it involves the spinothalmic fibres from the contralateral side. It can be localised at operation under local anaesthesia but this precludes its use in the seriously ill. Here, the use of evoked potentials is far better than for any other system of mixed nerves. Using evoked potentials and combining the operation with upper cervical posterior root section can give good pain relief in cancer of the face and throat.

\section{Mid-brain Tractotomy}

This operation, which was intended to interrupt the spinothalamic tract at its highest level in the dorsolateral tegmentum, has been virtually abandoned because of the high incidence of post-operative dysaesthesia and also the risk of deafness if the operation is done bilaterally.

\section{Stereotactic Mesencephalotomy}

This has some advantages in that a very small lesion can be made very accurately and its aim is to 
include the more medially placed palaeo-spinothalamic fibres. Its complication is a very high risk of diplopia and it cannot be done bilaterally because of the risk of hearing loss. It is a procedure with limited application and is done only at a few centres where particularly interested surgeons perform it (Nashold et al. 1969).

\section{OPERATIONS ON THE THALAMUS}

Despite more than 20 years of activity in this field, there is little indication that a single operation, or even a multicentric operation in this region, will give pain relief devoid of complications. There is a fairly high risk with a reasonably low level of success. It is as yet no more than experimental surgery, best suited to a limited number of centres and not universally applicable. It is of very little use in the treatment of thalamic pain and for other conditions in which it has been tried. Better methods of treatment are available.

\section{OPERATIONS ON THE FRONTAL LOBE}

There was a time when it was thought that ablation of parts of the frontal lobes would solve the problem of suffering associated with pain. Prefrontal leucotomy interrupts the frontothalamic projection system, and emotional responses are affected more than intensity of pain experience because there is no division of fibre tracts directly responsible for the carrying of impulses concerned with nociception. To be effective, these operations have to be rather extensive and then carry an unacceptable risk of personality disturbance. When they are of smaller format, they fail in their purpose of pain relief. The advent of various psychotropic drugs has made the use of these operations very limited indeed. In some very selected individuals with pain, this type of operation, particularly the very limited operation of cingulotomy, may play a part. A patient with an obsessive compulsive personality who now has the burden of being morbidly preoccupied with his plight could perhaps be helped.

\section{ELECTRICAL STIMULATION}

Electrical stimulation can be effective in controlling pain, even if severe and persistent, although appreciable difficulties and limitations still exist. Effective pain control methods are transcutaneous, percutaneous, peripheral nerve, dorsal column, ventral column, thalamic and internal capsular stimulation. These methods vary in regard to risk, effectiveness in given pain problems, duration of benefits and technical difficulties. The enthusiasm for brain stimulation methods has somewhat decreased almost as rapidly as they have evolved. The technical problems of electrode failure, electrode movement and the variability of human anatomy are measureable defects. The difficulties with patient selection still seem to reign supreme and the success and failure rates run parallel to all other methods of pain treatment. Time is necessary to assess the long-term results of treatment and effects on the brain. It has a limited application in carefully selected patients and this should be done at special centres.

The answer to pain is not surgery. It is doubtful whether the answer will be electrical stimulation because it is based on the same shaky anatomico/physiological assumptions as surgery. The answer will most likely be biochemical and at the as yet nebulous plane of interaction between chemical action and psychological experience.

\section{References}

Abbe, R. (1889): A contribution to surgery of the spine. Med. Rec. N.Y. 35, 149 - 152.

Maher, R. M. (1955): Relief of pain in incurable cancer. Lancet 1, 18 - 20.

Mullan, S., Hekmatpanah, J., Dobben, G. and Beckman, F. (1965): Percutaneous intramedullary cordotomy utilizing the unipolar electrolytic lesion. J. Neurosurg. 22, $548-553$.

Nashold, B. S., Wilson, W. P. and Slaughter, D. G. (1969): Stereotactic mid-brain lesions for central dysaesthesia and phantom pain. J. Neurosurg., 30, $116-$ 126.

Sjöqvist von O. (1937): Eine neue Operationsmethode bei Trigeminusneuralgie durch Schneidung des Tractus Spinalis Trigemini. Zbl. Neurochir. 2, 274-281.

Spiller, W. G. and Martin, E. (1912): The treatment of persistent pain of organic origin in the lower part of the body by division of the anterolateral column of the spinal cord. J. Am. Med. Ass. 58, $1489-1490$.

Sweet, W. H. and Wepsic, J. G. (1974): Controlled thermocoagulation of the Trigeminal ganglion and rootlets for differential destruction of pain fibres. Part I: Trigeminal neuralgia. J. Neurosurg. 40, 143 156.

\title{
THE ATTITUDE OF THE MEDICAL PROFESSION TO CHIROPRACTIC +
}

\author{
J. N. DE KLERK* M.B. Ch.B. (U.C.T.), F.R.C.S. (Edin.)
}

\section{HISTORICAL BACKGROUND}

The philosophy underlying chiropractic was first propounded by Daniel David Palmer in the city of

* Head, Dept of Urology, University of Stellenbosch and Chairman, Federal Council, Medical Association of South Africa.

† Address given to physiotherapists and students as part of Congress and 13th National Council in Cape Town, 27th April 1979.
Davenport, U.S.A., in 1895. Palmer claimed that the secret of all disease had been revealed to him and it was caused by displaced vertebrae which pressed against nerves. "By displacement and pressure, they elongate the pathway of the nerve in a manner similar to that by which an impingement upon a wire of a musical instrument induces it to become taut by displacing it from a direct line. This pressure upon a nerve creates greater tension, increased vibration and consequently an increased amount of heat. Heat alters tissue; altered tissue modifies transmission of impulses; modified impulses cause function to be performed 